Rhode Island College

Digital Commons @ RIC

\title{
Exploring Nurses' Attitudes About the Value of a Hospital Based Medical Futility Policy
}

Christie M. Bowser

Rhode Island College

Follow this and additional works at: https://digitalcommons.ric.edu/etd

Part of the Other Nursing Commons

\section{Recommended Citation}

Bowser, Christie M., "Exploring Nurses' Attitudes About the Value of a Hospital Based Medical Futility Policy" (2016). Master's Theses, Dissertations, Graduate Research and Major Papers Overview. 141.

https://digitalcommons.ric.edu/etd/141

This Major Paper is brought to you for free and open access by the Master's Theses, Dissertations, Graduate Research and Major Papers at Digital Commons @ RIC. It has been accepted for inclusion in Master's Theses, Dissertations, Graduate Research and Major Papers Overview by an authorized administrator of Digital Commons @ RIC. For more information, please contact digitalcommons@ric.edu. 


\section{EXPLORING NURSES' ATTITUDES ABOUT THE VALUE OF \\ A HOSPITAL BASED MEDICAL FUTILITY \\ POLICY}

by

Christie M. Bowser

A Major Paper Submitted in Partial Fulfillment

of the Requirements for the Degree of

Master of Science in Nursing

in

The School of Nursing

Rhode Island College

2016 


\begin{abstract}
Medical futility is a concept that emerged in the 1980s and despite multiple approaches to define the term, no specific definition has been socially accepted. For the purpose of this research, futility was defined as "treatment or clinical interventions that are not likely to result in benefit to the patient or produce the expected outcome" (Terra \& Powell, 2012, p.103). Consistently, there are disagreements between patients, family, and healthcare providers when it comes to end of life care. Families sometime pursue all available interventions in order to keep their loved one existing in any state of being, often times going against the wishes of the patient (Burns \& Truog, 2007). The purpose was to explore nurses' attitudes supporting a hospital-based futility policy. A futility policy gives the hospital and ethics committee more authority in the recommendations of the ethics committee. A qualitative descriptive design was utilized with face to face interviews of nine graduate nursing students. The participants were all female with varying years of nursing experience, matriculated in a graduate nursing program at Rhode Island College. Regardless of experience, all the participants were familiar with the term futility. For most of them, the most memorable patient they cared for was one who had unnecessary medical treatment at the end of life. Many were frustrated with the amount of money and resources utilized on futile medical treatment. Most of the nurses were in favor of working in a facility that had a futility policy because such policies have the capacity to decrease moral distress and assist healthcare providers in advocating for their patients. Ethics committees are also useful in providing healthcare professionals with needed support in end-of-life cases by exploring all points of view in the care and facilitating communication between the healthcare team and the patient and family. Recommendations and implications for the advanced practice role are discussed.
\end{abstract}




\section{Acknowledgements}

I would like to thank my supportive friends and family who were always there for me when I needed to vent my frustration about graduate school, and especially this research paper. It was a lot of work, but in the end, I can appreciate its value for it is one of my greatest accomplishments. I would like to especially thank my husband for always believing in me and being my biggest cheerleader throughout life.

I am thankful for my three readers; Dr. Annette Griffin, MSN, RN, MBA, Dr. Cynthia Padula, PhD, RN, CS, and Dr. Angela Creta, DNP, RN, CNL, NE-BC. I am in awe of the hours of work you have put in editing this paper, and am grateful for all the support you have given me along the way.

I would also like to thank my peers, specifically Lindsay Mook, who is always rooting for me and encouraging me to pursue my goals. I would have never made it through graduate school without the group texts with Jessi Corrente, Ashlee Viveiros, and Carly Shields-Pirri. You ladies are the best, it's amazing what we can do when we support each other! 


\section{Table of Contents}

Background/Statement of the Problem ............................................................................

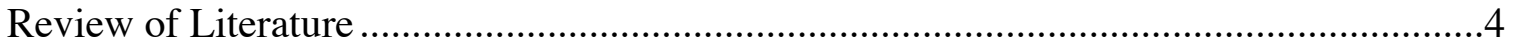

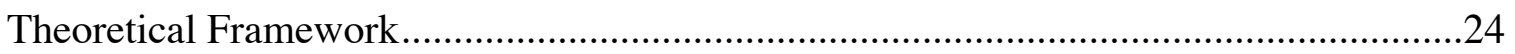

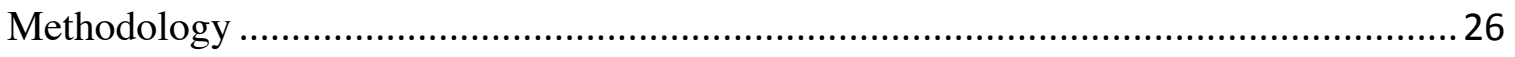

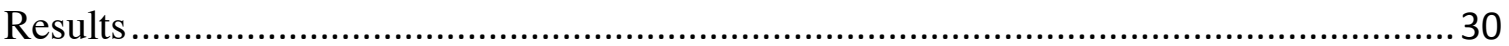

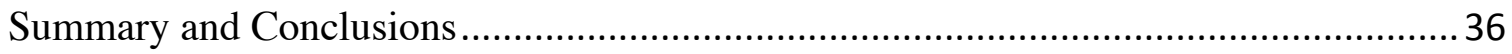

Recommendations and Implications for Advanced Nursing Practice ............................ 43

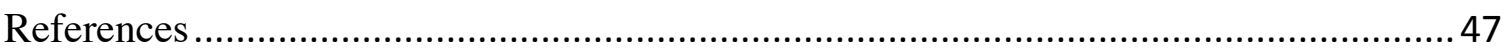

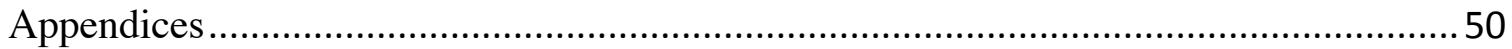


Exploring nurses' attitudes about a hospital based futility policy

\section{Background/Statement of the Problem}

Nurses are responsible for providing care for patients and following through with a physician's orders, however, it is not always easy to do. Certain cases are more difficult than others, especially those involving end-of-life care versus continuing with life-sustaining measures. It is not an easy decision for families or patients to make, but sometimes these life-sustaining interventions can do more harm than good, causing much distress among caregivers (Burns \& Truog, 2007). Some cases have become hallmark cases like that of Terri Schiavo, a woman who was in a persistent vegetative state from 1990 to 2005 while her parents and husband fought between keeping her alive or letting her go peacefully (Quill, 2005). Another difficult case in 2007 involved a toddler, Emilio Gonzales, whose mother fought for more time with her child rather than removing the ventilator (Cohen, 2007). Refer to Appendix A for an exemplar that occurred in a

hospital without a futility policy and inspired the writer to research nurses' perceptions of a futility policy.

There are two major historical events that shaped the future of healthcare decision making, both occurring in 1990. The first is Cruzan v. The Missouri Department of Public Health, which was the first case of a patient fighting over her right to die that made it to Supreme Court. The results of the case ruled that states have the right to establish standards of proof for advance directives, competent adults have the right to choose or refuse any medical or surgical treatment, adults can make advance directives and appoint a surrogate decision maker, and the surrogate can choose or refuse treatment on behalf of the patient. Following the case, the Patient Self Determination Act (PSDA) was soon established, which provides people with the opportunity to appoint a surrogate for end of life decision making (Rushton, Kaylor, \& Christopher, 2012).

When required to provide care that could be deemed futile to a patient, nurses often experience moral distress. For the purpose of this study, the definition of moral 
distress is when one knows the ethically sound action to take, but that action cannot be taken (Epstein \& Delgado, 2010). Intensive Care Unit (ICU) nurses report feelings of helplessness, hopelessness, guilt, anger, powerlessness, and frustration. Some feel that they have failed the patient (Whitmer, Hurst, Prins, Shepard, \& McVey, 2009). The most common ethical conflict nurses in the ICU described was "harm/good of life-prolonging aggressive therapies" because it was "prolonging the living/dying process with inappropriate measures" (Jacobs \& Taylor, 2005, p. 289).

Non-maleficence, the obligation to not intentionally cause harm, is a basic ethical principle that guides healthcare. Providing aggressive care can at times contradict the role of the healthcare provider. Researchers of futile care have shown that nurses are more affected with moral distress than physicians. One contributing factor is that nurses are in close proximity with patients and witness the suffering, therefore increasing the potential for their moral distress. Nurses are bound by nursing's Code of Ethics and the policies and procedures in the facility in which they are employed (Mohammed \& Peter, 2009).

Defining futility and its role in healthcare has been an ongoing issue since the 1980s. For the purpose of this research paper, futility is defined as "treatment or clinical interventions that are not likely to result in benefit to the patient or produce the expected outcome" (Terra \& Powell, 2012, p. 103). Nurses can suffer from moral distress when they are obligated to provide life-sustaining interventions to patients that have a small chance of benefiting from them. Nurses may experience less frustration and moral distress if hospitals implement a futility policy that provides guidance in these difficult cases. The purpose of this study is to explore nurses' attitudes regarding a hospital-based medical futility policy.

Next, the review of the literature will be presented. 


\section{Review of the Literature}

A search of the literature was completed through OVID documents and Google Scholar. Keywords of "medical futility", "futility policy", "rationing", "ethics committee", and "moral distress" were utilized. The search yielded 100s of results, which were selected by pertinence to the purpose. The final sample of literature that informed this study was 18 articles. Articles relating to pediatrics were excluded. The search was refined to focus on "nurses” and "nurses' perception.

\section{Futility}

Society, legislation, and medical teams are able to recognize there are moral and ethical reasons to limit medical interventions even though such interventions are available. Medicine can delay death, however, it does not always cure or have the ability 
to reverse the dying process. The best interest of the patient is not always aggressive care; which at times can be cruel or inhumane (Mohammed \& Peter, 2009). An example is a patient over the age of 85 being treated aggressively with chemotherapy. The patient's organs are aged and not functioning well, then the patient has to cope with the side effects of chemotherapy and endure further organ damage. The patient ends up suffering with nausea, vomiting, pain, etc. while life may not have been prolonged, just decreased in quality. There are cases where the disease takes over the patient, and medicine is ineffective. An example of this is Acquired Immunodeficiency Syndrome (AIDS). The patient has lost all capabilities of fighting opportunistic infections and the course of the disease comes to the point where despite all medical treatment available, the patient will die of the disease process. Futile care does not uphold the moral integrity of healthcare. The purpose of healthcare is to preserve health, prevent disease, treat disease, and encourage health promotion activities, not cause pain and suffering at the chance to increase quantity of life (Mohammed \& Peter).

Nurses have ethical responsibilities to uphold, as stated in the American Nurses Association Code of Ethics (2015). Nurses treat all patients regardless of individual differences. Care provided by the nurse is respectful to the dignity and worth of the patient. Nursing care aims to maximize the values that a patient treasures in life, and the care extends to support the family. Nurses act as advocates to the patients, ensuring that interventions are appropriate and necessary in order to minimize patient suffering (American Nurses Association).

Medical futility is a fairly new concept that arose in the 1980s. Families were demanding life sustaining procedures that healthcare providers felt were inappropriate. Limiting such life prolonging efforts were viewed by healthcare providers as an anticruelty policy, however, patients and families disagree. The concept of medical futility is still debated. Burns and Truog (2007) stated that the current debate of futility needs to be individualized. An effective plan of care begins with clear and honest communication 
between the healthcare team and the client to ensure all the information is presented in order for the most appropriate decision to be made (Burns \& Truog). Futility lies between the authority of the healthcare provider to limit harmful interventions and patient autonomy (Mohammed \& Peter, 2009).

The definition of futility is subjective and expands the realms of clinical, ethical, moral, religious, spiritual, and legal thoughts, beliefs, laws, values, and policies (Jacobs $\&$ Taylor, 2005). The term futile not only is difficult to define, but it has negative overtones. Some medical professionals and ethicists advocate that the term futile should not be used with family. In place of futility, the term medically inappropriate can be used to clearly define treatment that is considered appropriate by a medical professional. It also helps to clarify what appropriate treatment consists of. Why a medical intervention is considered to be ineffective needs to be made clear to patient and families; it promotes better understanding (Wilkinson \& Savulescu, 2011). There are incongruences with defining futility, a lack of accepted procedural approach, and issues with conflict resolution and negotiation (Burns \& Truog, 2007).

Families sometimes feel that physicians deem care is futile simply because they do not want to continue care. Families are apt to voice their feelings about physicians' decisions to the nurses. In order to satisfy the patients and families, physicians will often order the aggressive interventions the family would like to pursue. Nurses are then faced with having to support the family while providing care that is ordered by the physicians even when the nurses disagree with the aggressive level of care. Conflicts arise because physicians and nurses have the duty of non-maleficence and families have autonomy (Whitmer et al., 2009).

Different religions may have opposing views on whether life should be prolonged. For example, Catholicism views a feeding tube as an appropriate life prolonging measure for a person in a persistent vegetative state because all life, regardless of quality, is sacred. Buddhists believe that life should not be preserved at all costs, and if someone is 
dying, there is no need to go to extraordinary lengths. According to the Council on Ethical and Judicial Affairs of the American Medical Association, "a fully objective and concrete definition of futility is unattainable" (Jacobs \& Taylor, 2005, p. 289).

In order to better define futility, different concepts are utilized. According to Burns and Truog (2007), the first generation of the futility debate dates back to 1990 with Dr. Donald Murphy and Dr. Thomas Finucane. These doctors proposed seven clinical conditions in which further treatment should not be warranted based upon past case studies. Some examples are coma lasting greater than 48 hours and Human Immunodeficiency Virus (HIV) infection with more than two episodes of Pneumocystis Pneumonia (Burns \& Truog). Within the same year, Dr. Schneiderman, Dr. Jecker, and Dr. Jonsen began defining futile care based upon if the past 100 cases interventions were ineffective. These examples of past research yielded three concepts of futility: qualitative, quantitative, and physiologic (Burns \& Truog).

In qualitative futility, the risks do not justify the benefits. The goals of the patient or family may be viewed as unreasonable (Mohammed \& Peter, 2009). Qualitative futility refers to the state in which the quality of life after the intervention is remarkably poor (Whitmer et al., 2009). The hallmark case of Terri Schiavo, is an example of a woman who remained in a persistent vegetative state for 12 years due to disagreements between her parents and husband. A feeding tube was placed in order to keep her alive until the court ruled to have it removed in 2005 (Quill, 2005).

Quantitative futility is described as the chance that success is remarkably poor. An example of this is the personal exemplar in Appendix A. The patient was refusing dialysis based upon quality of life. She did not want to spend four hours, three times per week in dialysis nor feel the fatigue after dialysis in order to prolong her life. She preferred to be comfortable and live what time she had left.

Physiologic futility is a concept in which procedures are deemed futile if they are not able to meet physiologic goals. A physiologic goal is measured as a lab value, for 
example, mechanical ventilation achieving blood gases that are life-sustaining (Burns \& Truog, 2007). One issue with physiological futility is that data on many treatments are not available. There is rare evidence suggesting an intervention will fail $100 \%$ of the time, leading to doubts in stopping treatment (Mohammed \& Peter, 2009). Each case has different variables impacting the ability to predict that an intervention will be effective or ineffective. At times, a healthcare team may predict that a patient will not wake from a coma or a patient will never walk again, but despite the $<1 \%$ chance, the patient is able to recover (Mohammed \& Peter).

Jacobs \& Taylor (2005) argued that the healthcare team may use their experience to prognosticate futility. If a treatment proved to be useless in the last 100 cases of a healthcare provider's experience, then, in accordance to the principle of probability, the intervention should be judged as futile. Medical professionals and ethicists argue that an intervention should be deemed as futile if it "merely preserves permanent unconsciousness or fails to end total dependence on intensive medical care" (288). There are many flaws with defining qualitative, quantitative, and physiologic futility. Each case must be individualized, and it is impossible to define futility for every disease process and difficult to definitively prognosticate. Some families may agree that chances of $<1 \%$ success are worth taking. In many cases, families argue that life in a persistent vegetative state is still worth living. Society as a whole has not come to an agreement on this assumption (Burns \& Truog, 2007). In contrast, Whitmer et al. (2009) defined medical futility on a continuum. The sanctity of life, believing that life is sacred and all measures to preserve life are suitable is an example of one end of the spectrum. On the other end is quality of life, which is based upon individual beliefs (Whitmer et al., 2009).

Honeybul, Gillet and Ho (2013) described a more contemporary model of futility. They described futility in terms of the substantial benefit and risk of unnecessary harm for a more patient-centered approach. These terms are used to better individualize the term futility to incorporate religious, cultural, and social values. Substantial benefit 
describes an outcome that would be worthwhile to the patient, whether now or in the future. The risk of unnecessary badness is the possibility that the patient will end up in a state that he or she believes to be intolerable (Honeybul, Gillett \& Ho). 


\section{Futility Policy and Law}

Few hospitals have futility policies nor do many states have futility laws. Efforts to develop a procedure to resolve futility debates are hospital and state-dependent (Burns \& Truog, 2007). One example of a hospital futility policy is Boston Children's Hospital Futility Policy (Appendix E). When repeated efforts of agreement on treatment have failed, the futility policy is utilized. The policy is bilateral, meaning the healthcare team can consult the ethics committee when they believe care is futile and the family wishes to continue treatment or the family can consult the ethics committee if they believe treatment should be stopped and the physician wishes to pursue treatment. All parties involved are given the opportunity to discuss the case in front of the ethics committee. If the committee rules in favor of the clinicians, the hospital has four options. First, it can ask for further negotiation with the family. Second, it could transfer the patient to a facility that is willing to provide treatment as the family wishes. Third, the court can appoint an alternative decision-maker. Fourth, if none of the three approaches are successful, the hospital can invoke unilateral withdrawal of life support after giving the family or surrogate of the plan an opportunity to pursue legal advice. The Boston Children's Hospital policy has influenced some hospitals nationwide, that have adopted a facility based futility policy. A futility policy gives the hospital and ethics committee more authority in the recommendations of the ethics committee. If the ethics committee feels that care is futile and should be stopped, without the policy, this only stands as a recommendation and does not have to be implemented. Ethics committees make recommendations that can be strengthened when there is a medical futility policy in place. Some areas that have adopted futile care policies besides Texas are Iowa and California (Burns \& Truog).

A similar guideline policy from 1999 was by the San Francisco Bay Area Network of Committees (BANEC). The guideline defined non-beneficial treatment, allowed physicians to determine what constitutes non-beneficial treatment, and created a 
procedure to address when physicians and families disagreed. The treating physician was required to consult another physician. If both physicians were in agreement, they were to approach the family. If the physicians disagreed, the case was brought to the ethics committee. If the ethics committee disagreed with the primary physician, the patient could be transferred to a different physician or a different facility. If the ethics committee agreed with the primary physician, the family would be informed. The family could either withdraw treatment or obtain a court order for transfer to another facility. The policy was adopted by many hospitals in the Bay Area, however, there is no documentation of whether the policy was enforced or effective (Luce, 2010).

Some states have taken a procedural approach to the level of legislation. The procedural approach is imperfect because it may not appropriately differentiate between futility and rationing. Futility is based on whether or not an intervention will work whereas rationing, is whether the intervention is worth trying. Futility policies should never be utilized in an attempt at cost-containment (Burns \& Truog, 2007).

Texas enacted the Texas Advance Directives Act, which is similar to the Boston's Children's Hospital futility policy, but also requires a 10-day waiting period before the withdrawal of care if the ethics committee deems care to be futile. The authority for resolving the dispute between family and clinicians lie in the hands of the ethics committee. The committee is always available and is experienced in resolving these issues. If the family disagrees with the decision made by the ethics committee, it is difficult for them to dispute it. The family can bring the ethics committee to court, however, the court generally rules in favor of the ethics committee due to the Texas Advance Directives Act (Burns \& Truog, 2007).

It is common for ethics committees to conclude that disagreement between families and clinicians generally stem from miscommunication. Explaining the medical aspects of a critically ill patient with cultural sensitivity is difficult and requires expert communication skill not learned in medical school, which sometimes results in giving 
family a sense of false hope. Most critically ill patients are unable to participate in endof-life decision-making, therefore, family is put in the position to speak for the patient (Burns \& Truog, 2007).

Physicians are believed to have the ability to predict whether or not an intervention will accomplish a goal, however, this is not always the case. Studies regarding futility have shown that families are less satisfied with care when aggressive treatment is pursued. Evidence-based practice guidelines recommend that nurses discuss and allow the patient to set his or her own goals in care (Nolger, 2013). Recently, a Physician's Order for Life Sustaining Treatment (POLST) form has emerged to provide the patient with more autonomy in end of life care. Originating in Oregon in 2012, the POLST is a specific form addressing cardiopulmonary resuscitation (CPR), nutrition, hydration, intubation, and comfort care measures. The purpose of POLST is to assist in end-of-life care by improving communication between patients and caregivers, encourage shared decision-making between patient and caregiver, and ensure that a patient's wishes are honored. Different from an advanced directive, it should only be utilized for patients with serious illness and probable death within one year. Because POLST is organized and specific, nurses are able to use it to begin setting goals of care with the patient. It provides nurses with standing medical orders. While advanced directives are guidelines for future care if something were to happen, POLST addresses current medical care. Currently, the states that have adopted POLST are Oregon, California, Washington, Idaho, Montana, Utah, Colorodo, Louisiana, Tennessee, North Carolina, Georgia, New York, Pennsylvania, and West Virginia. Most other states across the United States are developing a POLST program (Nolger).

The concept of futility encourages physicians to use their judgment and empirical evidence to determine if treatment is appropriate, which affects the way the information is presented to the patient and family. The presence of futility gives physicians the right to recommend or withhold interventions. The term futility can be literally defined as 
incapable of being accomplished. The Acute Physiology and Chronic Health Evaluation (APACHE) is a number scoring system that was established to promote objectivity in determining futile care. In addition, the APACHE was developed due to the frequency of poor outcomes of ICU admissions, the high cost of treating them, and the shortage of ICU hospital beds. Physicians who created the APACHE were guided by the ethical principles of distributive justice, beneficence, and non-maleficence. The scoring systems proved to have both positive and negative predictive abilities, therefore, only remained an adjunct in the determination of futile care. Since its development, it has been often used in ICUs across the United States (Luce, 2010).

There are several options available for resolving futility disputes. Capitation is defined by Wilkinson \& Savulescu (2011) as providing treatment that a patient or surrogate wants even though it is futile. Another option is that the clinician can unilaterally withdraw care from the patient. The clinician can seek consultation from the ethics committee. The healthcare team could request appointment of an alternate surrogate. Finally, settlement from the court can be requested (Wilkinson \& Savulescu).

Discussions about medical futility often disregard the factors of resource allocation. Non-clinical factors, such as cost, age, socioeconomic status, or gender, is socially unacceptable as determinants to medical futility. The concept of utilitarianism holds that medical care should not be offered if the desired effect is absent or limited, thus conserving resources for those who will benefit from them (Terra \& Powell, 2012).

Medical futility can be viewed from the perspective of distributive justice. Prolonging the dying process is a huge cost to society. A valid argument against distributive justice is that physicians do not function as cost-containment agents. It is preferable that society set guidelines or pass laws to limit futile medical treatment, however, these guidelines fuel the debate of rationing (Whitmer et al., 2009).

Concerns arise due to the fact that the aging population is growing in size. Healthcare costs continue to increase annually. The way resources are used or allocated 
will be an issue that continues to develop as healthcare evolves (Mohammed \& Peter, 2009). Re-admissions are another source of healthcare costs. There are many patients who could, or should, be utilizing palliative care, but instead are being constantly readmitted to acute care (Terra \& Powell, 2012). Enacting a futility policy could potentially decrease readmissions and potentially improve end-of-life care. Rather than having frequent readmissions, poor pain control, false hope that the plan of care will lead to a cure or increased length of life, the patient and family will benefit from the support from proper end-of-life care.

Healthcare professionals and family members know that death has occurred when respirations and heart beat cease. Society is in much debate over the concept of what defines human life. One major issue is that there is an increase in the number of unconscious ICU patients. Some people consider human life to be a state of consciousness, and believe an unconscious patient may benefit from life-sustaining measures. The unconscious patient may be sustained on life support for many years, therefore a time limit may need to be enforced on the use of life support for the unconscious (Whitmer et al, 2009).

\section{Healthcare Decision-making}

Autonomy has been established as the dominant ethical principle in America. Physicians participate in shared decision-making with families via informed consent. In the past, hospitals in America practiced a universal resuscitation policy. Currently, physicians are required to ask patients their wishes regarding resuscitation upon admission. Patient autonomy limits a physician's ability to decide to treat or not to treat (Luce, 2010).

Clark (2010) noted that patients in ICU feel safe when family members are present and rely on their family members to make the right decisions for them. In this study, the patients felt as though they are being watched over, comforted, and encouraged. It is important that families be present for invasive procedures because it 
provides them with active participation in care. Families serve as the gap between the patient and healthcare providers. The active participation of families decreases the incidence of ethical dilemmas (Clark).

Treatment decision-making has shifted from the healthcare provider to giving the patient and family more autonomy. The decision-making ability has allowed patients and families to request aggressive treatment whether or not the illness is curable. Treatment options are allowed without regard to prognosis. It is challenging for healthcare providers to balance the patient's best interest with the request for advanced treatments (Mohammed \& Peter, 2009).

Surrogates are often hesitant to believe the predictions of the physician for a variety of reasons including decreased confidence in the provider from prior experiences of inaccurate prognosis, the need to see for themselves whether the patient is capable of recovery, the need to seek more information, and religious beliefs. Not all aspects of life are understood with absolute certainty. Generally, surrogates have misunderstandings about prognosis or have religious-based objections. It may be appropriate to override a surrogate's religious beliefs that conflict with a physician's prognosis (Zier et al.,2009).

\section{Nurses' Beliefs on Futility}

Sibbald, Downar, \& Hawryluck, (2007) studied different healthcare providers’ perception of futile care in the intensive care unit. The study used semi-structured interviews. The researchers interviewed 14 physician directors, 16 nurse managers, and 14 respiratory therapists from 16 ICU's across Ontario. The responses were analyzed using a modified grounded-theory approach. It was found that generally, nurses are less satisfied with end-of-life decision-making when compared with physicians. Out of the team members, nurses are usually first to feel that life-support should be withdrawn from a patient, which can lead to conflict with physicians. Physicians often offer treatment to patients with a poor prognosis, giving the patient and family false hope. The study also 
concluded that nurses often predict mortality more accurately than physicians (Sibbald et al.).

Ferrell (2006) studied the impact providing futile care had on nurses in the ICU. She interviewed 108 nurses and obtained narratives from each of the nurses. Nurses described care as "torture," "violence," and "cruelty" (p.923). Physicians would round on their patients in the morning, promising the patient and family yet another aggressive treatment option, and the nurses would spend another day providing futile care to the patient. The nurses began to exhibit feelings of anger, frustration, and symptoms of moral distress. Nurses believed that there is a huge social injustice when patients are denied basic healthcare while others have access to costly futile care. Another source of moral distress was a patient and family's religious belief that a miracle will happen, which clashes with beliefs of modern medicine causing more frustration. With the many sources of moral distress inflicted by futile care, nurses are not left with much support (Ferrell).

\section{Role of the Ethics Committee in Futility Cases}

The purpose of ethics committees is to provide support to clinicians, reduce moral distress, and aiding clinicians to maintain ethical practice. Ethics committees emerged in the 1960s, utilized as review boards for abortions. Then by the 1970s, their use focused shifted to deciding which patients were going to receive hemodialysis due to a machine shortage. They were also used in decision making to withdraw care from handicapped infants. As demonstrated by their function, ethics committees were utilized to protect the healthcare organization. By the 1990s, ethics committees were mandated to focus on ethics rather than legal issues. The Joint Commission mandated a standard that hospitals have an ethics committee (Lachman, 2010).

The functions of the ethics committee are policy review and development, case consultation, and ethics education. Some typical policies that an ethics committee would review are care of the dying patient, informed consent, code status, life-sustaining 
treatment, and decision-making for the incompetent patient. Continuing education is done by the ethics committee via article reviews and discussions, case studies, workshops, and member sharing (Lachman, 2010). Staff education is also provided by the committee through the same mechanisms listed above. The purpose of an ethics case consultation is to address ethical issues in an active clinical case. An ethics consult can be called by the patient, family, or any discipline; nurse, social work, pharmacy, rehabilitation, or physician. The committee members complete a 1:1 interview with the medical professionals caring for the patient, the patient (if possible), and caregivers. The medical record is reviewed. The problems are analyzed in terms of medical interventions, medical indications, patient's wishes, quality of life, and background information. After reviewing and debating the issues in the case consultation, the ethics committees provides recommendations and options for the medical team (Lachman).

An ethics committee needs to be made up of a diverse group of people to better explore various points of view. The participants of the ethics committee differ from hospital to hospital but may include nurses, doctors, theorists, retired physicians or other healthcare personnel, social work, chaplain, lawyer, etc. The purpose of the multidisciplinary ethics committee is to represent a diversified culture. Culture is a perspective that needs to be considered when ethical decisions are being made (Whitmer et al., 2009). It is important for the ethics committee to be culturally competent. Culture can be defined as any group of people sharing similar ideas, values, or beliefs. Cultural competence is essential to an effective ethics committee. Despite popular beliefs, culture is broader than race, religion, and ethnicity. Culture is a sum of beliefs and attitudes that distinguish one group from another. Healthcare providers represent a culture, but more specifically nurses have a culture of their own, which differ from the culture of the doctors. Healthcare providers must assess their own culture. It defines behaviors and norms for the human experience. The individual is the person's own preference, not 
necessarily consistent with cultural norms. Each individual member of the healthcare team provides further diversity due to his or her personal beliefs (Whitmer et al.).

The key points an ethics committee must consider in order to deem care futile include the defined goal of treatment and the patient's autonomy, welfare, and justice. In order to justify limiting or withdrawing treatment, the decision must be appropriate to the situation, it must accomplish the goal without causing unnecessary harm, and the outcome is probable by the agent(s) who have made the decision. For it to be rational, there should be a clear relationship between the decision, action, and result that the agent can explain. If the medical intervention is regarded as futile and the intent is to prevent harm, both justice and non-maleficence are achieved (Terra \& Powell, 2012).

\section{Role of Communication}

To aid in the prevention of ethical dilemmas, it is pertinent that healthcare providers utilize clear, consistent communication. Unfortunately, not all futility debates can be avoided even with perfect communication. It is essential that clear, consistent communication is continued through a conference involving the healthcare team, patient, and family. During the conference, the patient's condition, prognosis, and goals of treatment should be discussed. The patient and family need to voice their goals of treatment as well. At times, the patient and family request all life saving measures while the healthcare team disagree. The issue here may not be futility after all, but different goals of treatment (Whitmer et al., 2009). The healthcare team needs to be completely honest about the repercussions of life-sustaining treatments. For example, treating an elderly patient with chemotherapy for cancer can result in multi-organ system failure and death. Some physicians discuss code status and advance directives poorly and simply ask a patient if they are dying, do they want to be saved? This does not provide the patient with enough information to make an informed decision.

Families often may be under the assumption that their family member who survived an ICU admission with all life-sustaining measures will return to his or her 
baseline functioning. Unfortunately, this is often not the case. When life-sustaining treatments are performed on a patient, it is rare that the patient will return to baseline functioning (Whitmer et al., 2009). An example would be a patient who suffered cardiac arrest. During cardiopulmonary resuscitation in a hospital setting, some unfortunate outcomes may include brain death, broken ribs, medications can cause soft tissue damage if infiltrates, and loss of digits or limbs by medications that divert circulation to the heart and brain. Conversations with families need to include the residual disability after a serious illness or injury and realistic goals. Families may never take their loved one home. The patient may always be a patient, whether in and out of the hospital or at a long-term care facility (Whitmer et al.).

One of the most common beliefs is that the "miracles of modern medicine," and advanced technology can help people to avoid death. This belief stands in the way of quality end-of-life care. Healthcare providers may be evasive and avoid conversations with family regarding palliative options even when continuing care prolongs patient's suffering. Some families override the patient's advance directive or do not accept the poor prognosis. Healthcare providers need to be clear in communication and prevent the lack of understanding about patient care (Whitmer et al., 2009).

\section{Role of the Nurse}

Nurses have the ability and professional responsibility to advocate for their patients. Patients are more likely to express their wishes to their nurse. At times, the advocacy can be problematic because nurses are serving both patient and the physician (Mohammed \& Peter, 2009). The nurse is a mediator between the patient and the physician at times. Sometimes the nurse disagrees with the physician's plan of care for the patient, which puts in question what is the morally right thing to do for the patient. Nurses are often caught in the middle, causing more frustration (Mohammed \& Peter). Nurses in their role as patient advocates form trusting relationships with patients and families and often develop communication skills to mediate conflicts between the 
physicians and clients. Although, even with the best nurse advocacy and mediation as well as communication with the healthcare team, conflicts still arise. A change may be needed to address these conflicts, like a hospital policy or state statute. It is not acceptable to delay a conversation about futility and allow it to reach crisis level (Jacobs \& Taylor, 2005).

Nurses are on the front lines caring for patients, but are often left out of the decision-making process as far as plans of care. Nurses most affected by futility issues are those in critical care, hospice, and palliative care settings (Jacobs \& Taylor, 2005). Their job is extremely difficult because nurses form relationships with the physicians they work beside as well as relationships with the patients and families they care for. When the patient, family, and the physicians have opposing views, the nurse may have difficulty navigating between the feelings of both parties and remaining impartial. This group of strangers with different moral values are gathered together to decide the fate of the patient, whether it be quantity or quality of life, or death (Jacobs \& Taylor).

\section{Practice Implications}

Currently, the American Thoracic Society, Society for Critical Care Medicine, American College of Chest Physicians, American Association of Critical Care Nurses, and European Society for Intensive Care Medicine are jointly developing a guideline for medical futility in the ICU. There are two main aspects of the guideline. The first is to "first do no harm." Without offering meaningful benefit, the act of causing suffering is unacceptable. The second aspect is the precautionary principle, meaning "in the absence of clear data, if a policy has significant risk of significant harm, the burden of proof that it is not harmful falls on those recommending this policy" (Rubin \& Courtwright, 2013, p. 1708). The focus is on justifying unilateral decisions to limit or withdraw life sustaining measures despite the demands of the patient or family. The new guideline is to recommend a procedural approach to determine medical futility. The procedural approach offers a due process framework that considers a broader range of values, 
beliefs, and expertise. It will consider all possible interventions to determine if the risk outweighs the benefit. The Ethics Committee of the Society of Critical Care Medicine recommends that futility policies be discussed with and available to the general public, reflect acceptable moral values, be not completely reliant on prognostic scoring systems, incorporate a report of the appeals process, and are recognized by the court system. The American Medical Association suggests that if there is a disagreement regarding futility, healthcare providers should attempt to agree with family on what is futile care in advance, use joint decision-making to resolve conflicts, consult others to aid when conflicts arise, utilize the ethics committee, transfer the patient to another physician or facility, and if transfer is not feasible, the intervention should not be offered (Jacobs \& Taylor, 2005). Currently, the literature is being reviewed on how procedure-based futility policies function, the types of patients requiring futility evaluations, the ethics committee structure, and the clinical outcomes of futility judgments (Rubin \& Courtwright, 2013). This research study may help inform nursing practice about nurses' knowledge and perceptions regarding the implementation of a futility policy. Next, the theoretical framework will be discussed. 


\section{Theoretical Framework}

Quality of life means something different to everyone. Rosemary Rizzo Parse's Theory of Human Becoming addresses the meaning of quality of life to a patient and the impact it has on the nurse caring for the patient. A nurse often learns of a patient's perception of quality of life through structured and unstructured discussions, whether it would be providing daily care for the patient, hearing the family and patient interact, or in family meetings with the healthcare team. The nurse is usually attentive to changes in the meaning of life and is able to witness the patient's own value of living priorities. For the nurse to witness this, he or she is attending to the patient with unconditional presence. It is non-intrusive, and the nurse has the ability to reach beyond to honor the patient's human dignity in a non-judgmental manner. The nurse is able to embrace the patient's own unique values, and embraces the significance of the patient's choices. The nurse witnesses the patient's journey and is living true presence. This interaction is known as “coming-to-be-present", requiring preparation and attention (Parse, 1992).

The methodology of Parse's framework functions through illuminating meaning, synchronizing rhythms, and mobilizing transcendence. Illuminating meaning is defined as making things clear with speech, silence, and movement with rememberingprospecting, which is living in the meaningful moments of life. Synchronizing rhythms is engaging in the flow of connecting-separating with the human and universe, which is developing one's own rhythmic patter of living. Mobilizing transcendence is reaching out beyond oneself to thrust with the imagined possibilities of transforming all at once in the illuminating meaning moment (Parse, 1992).

The patient has to define his or her personal health description, which is the meaning of the situation, patterns with others, and his or her hopes and wishes. Patterns of becoming themes are communicated to the nurse. These patterns are what guides a nurse in caring for the patient. The nurse will learn the patient's intents and priorities, which are what the patient desires from healthcare providers and how it is addressed. All 
this information will help guide the nurse in focusing on the meaning of quality of life for the patient (Parse, 1992).

Parse's theory relates to this research because the patient and family are in control of healthcare decision-making. Their verbal and nonverbal communication and behaviors guide the nurse in caring for the patient. The nurse learns about the patient's beliefs and values. These beliefs and values guide how a nurse presents information and communicates to the patient and family. The nurse has to be willing to learn about his or her patient in order to better advocate for the patient. The knowledge the nurse obtains from the patient, influences how the nurse will react to how the family or physician opts for life-sustaining treatment. Having a hospital-based futility policy, may better support the nurse in caring for the patient when the patient no longer has capacity. The futility policy can better support the patient and family to guide in the decision-making process with the ethics committee.

Next, the methodology will be presented.

\section{Methodology}

\section{Purpose}

The purpose of the study was to explore nurses' attitudes regarding a hospitalbased futility policy.

\section{Design}

The study design was qualitative descriptive, utilizing open ended questions constructed by the researcher. A qualitative research design was chosen to better illustrate nurses' attitudes of a hospital-based futility policy. Moderately structured faceto-face interviews gave nurses the opportunity to speak freely about futility.

\section{Sample}

The population of Rhode Island College (RIC) graduate nursing students were mostly female of various age groups and nursing experience representing different cultures. All students in the Master of Science in Nursing program at RIC were included 
as potential participants regardless of race, age, or gender. The researcher anticipated a $20 \%$ response rate due to data collection occurring in the summer, conflicting with vacations and summer classes.

The participants of the study were graduate nurse students currently matriculated in the clinical nurse specialist, nurse practitioner, or nurse anesthetist program at Rhode Island College.

If greater than $50 \%$ of the participants who responded and were interviewed worked at the same facility, subsequent participants from the same facility were excluded. The rationale for excluding them was to allow for a more representative sample.

\section{Procedures}

This research was reviewed by the RIC IRB. With permission, an IRB approved e-mail was sent to 100 graduate nursing students along with an IRB approved informational letter explaining the study (Appendix B). Participants who were willing to

participate were asked to contact the researcher to set up a date and time for a face to face interview. The scripted email elicited a limited response despite repetition. Fellow students were informally encouraged to respond which increased participation. The researcher then encouraged peers to participate in the survey, which resulted in further interviews.

The researcher screened the volunteer for eligibility and then set up a time and date for the face to face interviews which were conducted on the Rhode Island College campus. The RIC campus was chosen because it is familiar to all students, provides a nonthreatening environment, and is convenient to students. Respondents were contacted one additional time prior to the interview to verify their intention to participate in the interview. Participants were informed that participation was voluntary, and they would be able to contact the researcher via email or phone in the event they had questions about the 
study or would like to withdraw from the study. Participants were informed that they may withdraw from the study at any time without penalty.

Immediately prior to the interview, the participants were asked to read and sign an IRB approved informed consent document (Appendix C). Participants were offered the opportunity to ask questions at this time. Responses to interview questions and observations of the respondents during the interview were recorded by the researcher. Answers to interview questions were verified for accuracy by reading them back to the respondent. Each interview took about 30-45 minutes. Interviews were continued until saturation was reached. Participants were entered into a raffle to win a \$25 Panera gift card.

\section{Measurement}

The interview questions were developed by the researcher from personal clinical experience and experience from being a member of a hospitalethics committee. The case study in Appendix A, from the researcher's nursing experience, brought forth some interesting concepts about futility and resource allocation. The interview included an explanation of what futility means, an example of a futility policy, and nine open-ended questions.

Initially, the researcher conducted a pilot to assess the interview questions for clarity and to estimate the time necessary to complete each interview. Two registered nurses (RNs) were interviewed and asked to comment on their perceptions of the questions, identify any questions that were unclear or difficult to answer and provide feedback on the length of time needed to complete the interview. Participants indicated

that there was a need for clarification of the definition of "rationing of healthcare" used in question three. In addition, clarification was also needed regarding the use of "moral distress in nursing". The interview time varied between 20 and 40 minutes between the two respondants. No other changes to the interview questions were necessary.

\section{Data Analysis and Storage}


The literature review did not yield a survey regarding questions about nurses' feelings about futility. Therefore, the researcher created the survey questions. The reliability and validity of these questions were discussed with the first reader faculty, then tested in the pilot as discussed above. The nurses interviewed gave the researcher feedback on the questions to ensure the participants had a clear understanding of what was being asked. After all the interviews were completed, the researcher and a fellow graduate nurse reviewed the data. Demographic data were illustrated in a descriptive summary, and data derived from the open-ended questions were sorted by question to examine for trend in the data. Data was then organized into relevant themes. The researcher reviewed the data by organizing the data by question and reviewing with the first reader faculty during a face to face meeting. The researcher read back the answers to the questions after recording them to confirm accuracy. The data was reviewed with an outside person. Faculty input was also utilized in order to confirm reliability and validity of the data. Data was stored in a folder labeled "Futility Interview Responses" and kept in a lock box in an office at the Yellow Cottage at RIC.

Results will be presented. 


\section{Results}

The researcher had anticipated that saturation would be reached at 10-20 interviews. Nine interviews, including the two pilot interviews and seven additional interviews were conducted, and saturation was reached. The sample was comprised of nine female nurses. The graduate nursing students worked at various hospitals in Rhode Island, which provided different patient examples than if the participants were from one particular hospital. The range of experience as a registered nurse ranged between less than one year to 27 years.

Overall, most nurses were poised and confident in their responses. However, the nurse with less than one-year experience seemed very nervous. The participant stated that she was not confident in the subject and was "afraid" to give a wrong answer. The researcher reassured her that there were no wrong answers because the questions were based on opinion.

The first question of the interview was to "Please describe to me your understanding of medical futility." Regardless of experience or confidence in answering the question, all participants were able to provide understanding of medical futility and describe relevance to their practice. Some examples are as follows:

"Any further medical treatment would not change the course of events."

"Medical futility is used to describe interventions that are unlikely to benefit the patient in any significant way."

"Medical futility is the continuation of using life sustaining treatments that are providing to be of no benefit to the patient, such as blood, treatments, etc. They will not improve the outcome for the patient."

The second question asked about experiences regarding futile care and how it affected the participant both personally and professionally. All participants were able to discuss some experience with futile care, even if it wasn't necessarily labeled that way in practice. One shared two of her own personal cases with futile care, her mother and best 
friend. Both her mother and best friend chose to stop treatment to increase the quality of the time they had left. The participant stated that in these cases she acted as a family member or friend, and separated herself as a nurse, but felt her medical knowledge and nursing experience helped her support their decisions. Both these experiences "inspired" her to become an oncology nurse, in which she continues to support families, aid in decision making, and have these end of life conversations about treatment versus no treatment. She thought that these experiences made her a better nurse.

Another participant believed that something usually happens to the patient to aid in the decision-making process, like a fall, infection, or worsening condition. These futile experiences made another participant realize "how close death is to all of us. It can happen anytime to anyone." A critical care nurse discussed that families have limited understanding of medical care and make decisions based on religious or cultural belief. She stated, "It is hard as a nurse to see a patient, with no hope of recovery, suffer for years immobile in bed, on a vent [ventilator], and receiving tube feeds while they develop painful infections, pressure ulcers, or other complications because the family refuses to let the patient go." Another nurse felt that futile care contributes to burnout in nursing. A nurse stated that other departments, like blood bank, will intervene in situations and refuse to grant blood products if a doctor orders it inappropriately. The facility also addressed life-sustaining treatment with families and encourage families to stop treatment if there is no benefit. Other participants voiced that it is difficult to see continued lifesustaining or invasive treatments when there is little or no hope in surviving.

The next question was comparing futility to healthcare rationing and participants were asked if they felt medical futility was the same as rationing healthcare. The responses were unanimous in that all the nurses disagreed that futility was the same as rationing. One stated, "I don’t think healthcare is rationed enough!" while another stated that "The amount of healthcare money spent at the end of life is outrageous." Another 
participant stated, "In practice, it's the opposite [of rationing]. Resources are poured in when death is near."

Participants were asked if the hospital in which they were currently employed has an ethics committee. All of the participants answered yes. The question was followed by asking about their experience with the ethics committee. With the exception of one participant, all had indirect to no experience with the ethics committee. The one member with experience was a former member of the ethics committee. She encouraged other nurses to utilize the ethics committee and attend the forums. Due to change in management, she stated that the ethics committee is phasing out. One participant stated that an ethics consult is avoided because the "culture is to speak with the doctor first." The participants were asked if they would feel comfortable requesting a consult. None felt comfortable and one even stated that she "wouldn't know where to start."

The next question focused on how a futility policy would function in a hospital and how it would affect nursing practice. Two people answered that it would provide support to nurses and the healthcare team. The nurses expressed that sometimes members of the team do not speak out about stopping treatment due to potential legal repercussions. One stated that a futility policy could be "dangerous territory" because every patient and every situation is so different. She did note, however, that if she were to utilize one, it would have to be mandatory for an ethics consult on these patients. One nurse was unsure what type of effect it would have because she had changed roles and was no longer a bedside nurse. Another felt as though medicine has "created an environment at times where the patient and their families dictate the standard of care," therefore a futility policy would struggle. Three participants stated they were supportive in a futility policy and felt as though it would be beneficial to the patient. One of them stated it would take "immense education" in order to get nurses and providers to utilize it because practitioners have a tendency to "always want to heal." Finally, one nurse stated 
that she felt it would not be utilized much, but perhaps could be contacted on smaller scale issues

The next question asked the participant if she was familiar with moral distress and if she felt comfortable to describe feelings about moral distress. Every nurse interviewed reported being familiar with moral distress. One nurse stated that after having an experience with a patient, family, and healthcare team, she actually "looked it up on [American Association of Critical-Care Nurses] AACN to learn more about it." She was glad know her feeling of helplessness had a name and that she was able to seek support. All the nurses were able to provide examples that they still think about; only one did not give an example involving medical futility. The patient she discussed was in his 20 's and refused a blood transfusion that could have saved his life. The nurse with less than a year experience had not encountered an experience causing her moral distress. All participants felt that moral distress occurred when the patient's decisions and values do not align with ours as healthcare providers.

The researcher then asked the participants if they believed if a futility policy would increase or decrease moral distress. All of the participants agreed that a futility policy would decrease moral distress. Participants stated that "it would have to be utilized correctly," "it would provide guidance," "decrease guilt in decision-making." One participant stated that she didn't feel like it would make a drastic change because “we are all human" and we can't take our feelings out of the cases we encounter.

The participants were given the futility policy from Boston Children's Hospital to review and share their thoughts on it. All participants were in favor of the futility policy when caring for the adult population. All stated that a presence of a futility policy would not deter them from working in a given facility. One participant reported being very bothered that the futility policy came from a children's hospital. She believed that children are a very sensitive subject when dealing with futility. She stated that as healthcare providers, "you want to give them [children] every opportunity to fight a 
disease or get better using any means you can.” One of the other nurses had a clinical rotation on a heart-lung transplant unit for children. She stated that she saw people from around the world just abandon their children on these units, knowing that they needed help but couldn't afford the medical costs. Some of these children were left to die. In these cases, the healthcare team had to do everything. Healthcare providers want to heal, especially children. Another nurse stated, "That policy seems like exactly what I would want to have in place at a hospital." One stated that she liked that it was bilateral, meaning the family could initiate it if they felt the healthcare team was choosing to overtreat the patient or the healthcare team could initiate it if the family was choosing overtreatment. She also liked that there was an option to transfer the patient.

Finally, the participants were asked how they felt a futility policy would work in a hospital and if it would change their nursing practice. Eight out of the nine participants felt a futility policy would function well in a hospital, as long as healthcare providers were properly trained in utilizing it appropriately. They felt that it would be a "resource" and "support" for healthcare providers, depending on how it's written and utilized. One nurse stated that "as a future [Nurse Practitioner] NP, I'm not sure that I'd be ready to utilize it" because she felt that families are entitled to a decision even if the healthcare provider feels it is wrong. One nurse felt that it wouldn't change things at all. She stated that it is much more powerful when family come to terms with the situation on their own and ultimately need to be the ones to decide when to stop life-sustaining interventions. Next, summary and conclusions will be presented. 


\section{Summary and Conclusions}

Nurses are at the frontlines caring for patients. Nurses may suffer moral distress when carrying out a plan of care for the patient that he or she does not agree with or it is against a patient's wishes. The purpose of this study was to explore nurses' attitudes about a hospital based futility policy. For this research, futility was defined as "treatment or clinical interventions that are not likely to result in benefit to the patient or produce the expected outcome" (Terra \& Powell, 2012, p.103). Medicine can delay death; however, it does not always have a cure or the ability to reverse the dying process. The best interest of the patient is not always aggressive care; which at times can be cruel or inhumane (Mohammed \& Peter, 2009). The concept of futility encourages physicians to use their judgment and empirical evidence to determine if treatment is appropriate, which affects the way the information is presented to the patient and family. Futility gives physicians the right to withdraw or withhold interventions (Luce, 2010). Discussions about medical futility often disregard the factors of resource allocation. The concept of utilitarianism holds that medical care should not be offered if the desired effect is absent or limited, thus conserving resources for those who will benefit from them (Terra \& Powell, 2012).

Medical futility can be viewed from the perspective of distributive justice. Prolonging the dying process is a huge cost to society. A valid argument against distributive justice is that physicians do not function as cost-containment agents. It is preferable that society set guidelines or pass laws to limit futile medical treatment, however, these guidelines fuel the debate of rationing (Whitmer et al., 2009). Sibbald, Downar, \& Hawryluck, (2007) studied different healthcare providers' perception of futile care in the intensive care unit. Out of the team members, nurses were usually first to feel that life-support should be withdrawn from a patient, which can lead to conflict with physicians. Physicians tend to offer treatment to patients with a poor 
prognosis, giving the patient and family false hope. The study also concluded that nurses often predict mortality more accurately than physicians (Sibbald et al.).

Most hospitals have an ethics committee to support the healthcare team with difficult cases, often involving end-of-life. An ethics committee helps to define goal of treatment and the patient's autonomy, welfare, and justice. In order to justify limiting or withdrawing treatment, the decision must be appropriate to the situation, it must accomplish the goal without causing unnecessary harm, and the outcome is probable by the agent(s) who have made the decision. For it to be rational, there should be a clear relationship between the decision, action, and result that the agent can explain. If the medical intervention is regarded as futile and the intent is to prevent harm, both justice and non-maleficence are achieved (Terra \& Powell, 2012).

The study was approved by the Rhode Island College IRB. The researcher developed open-ended interview questions derived from the key points derived from the literature review and past cases in clinical practice. An email was sent out to 100 matriculated nursing graduate students, who then contacted the researcher to set up a face to face interview. Informed consent was obtained prior to the nine interviews performed on the Rhode Island College campus. The researcher observed the behaviors of the participants, recorded the participants' responses, and read them back to confirm accuracy. Years of experience as an $\mathrm{RN}$ was the only demographic information obtained. The answers to the interview questions were sorted and read over by the researcher and a fellow graduate student to look for themes, similarities, and differences. The researcher consulted the first reader faculty to review the data in to look for trends.

All of the participants were female and type of employment included ICU, medical-surgical, emergency room, oncology, and case reviewer experience. Years of experience ranged from less than one to 27. Participants were able to describe an adequate understanding of medical futility. When discussing experiences regarding futile care, one of the participants was extremely comfortable discussing futile care. Her 
personal experiences with futility encouraged her to become a nurse in an area where futile care is prevalent. This participant noted that the patient and family's decision does not impact her personally and professionally, as long as they are making an informed decision. Another nurse stated that she completely sets aside her own beliefs and focuses on the plan of care for the patient, regardless of whether she agrees or disagrees. She takes time after caring for the patient to reflect on it. Nurses are taught to heal, however, sometimes healing isn't an option. In cases like that, it is the healthcare team's responsibility to aid in a peaceful transition. Interestingly, these two sets of answers are combining both perspectives. One supports the decision for further treatment, even if it's futile; the other advocates for a peaceful transition and an end to prolonged suffering. When speaking about rationing, all nurses agreed that there was a difference in futile care and rationing, despite the presence of this argument withinthe literature. In the state of Rhode Island, patients and families are potentially provided all the options when seeking treatment, regardless of social class, diagnosis, and resources. Ideally then, all patients are treated equally and are given individualized care. Patients who do not have a safe discharge plan may stay at the hospital until one is decided upon between the patient, healthcare team, and family or guardian. Patients and families are potentially granted the opportunity to make informed decisions, even if the healthcare team disagrees. At times this is frustrating, especially when patients choose partial treatment, as is illustrated in Appendix A. One of the participants gave an example on how a patient refused blood products due to being a Jehovah's witness, yet agreed to surgery in which he passed away less than ten minutes into the surgery. Some of the participants became frustrated about the amount of money spent at the end-of-life for futile treatment, recognizing that our healthcare system is in jeopardy due to the already high expense of healthcare. During the interviews, some of the nurses became very passionate when speaking on this subject because they felt that money was being wasted in treatments that caused pain and 
suffering to the patient, which caring for the patient was viewed as being very counterproductive.

The facilities in which the participants were employed had an ethics committee but it was disappointing to see how it was seemingly underutilized and how inaccessible it seemed for healthcare providers. Ethics committees are valuable in supporting the patient, family, and healthcare team. Some healthcare providers are not comfortable discussing end-of-life or stopping treatments and the ethics committee can be utilized for this. Ethics committees can also help to open up communication between patients and the healthcare team. In order for a futility policy to function in a hospital, it requires the support of the ethics committee. The healthcare providers would need ample education on utilizing a futility policy, as well as more awareness on when and how to consult the ethics committee. It would be essential for the ethics committee to be more involved in patient care in order for the futility policy to provide guidance to the healthcare team.

It was not surprising that all the nurses, regardless of experience, were familiar with moral distress. Even early in a career, moral distress is felt for many reasons. As the researcher expected, the participants discussed end-of-life cases that caused them moral distress. It is hard to watch a patient suffer or endure painful treatments with little hope for survival. After hearing the responses to this question, it was not unforeseen that most of the participants felt that a futility policy could decrease moral distress. When the participants were given the Boston Children's Futility policy to review, most of them were shocked that a children's hospital had a futility policy. In the way they answered the questions, it became clearthat they didn't support it for children, but supported it for adults. From their perspective, most participants thought a futility policy would be effective if used appropriately in the adult population. Of note, all of the nurses interviewed had not cared for children, which may contribute to their reservation of employing such a policy in this group. There did not appear to be a trend with years of experience and acceptance of a futility policy. It seemed that regardless of experience, 
nurses were accepting of a futility policy. For most participants, the source of moral distress in their careers was caring for patients who were continually treated with interventions that were not effective due to poor prognosis.

Some limitations included that only Rhode Island College graduate nursing students participated and they were all females. All were also in the final year of the Master's program; no students at other levels participated. It was difficult to set up interviews with students because of the need to meet on campus and time constraints associated with being a graduate student. More responses might have been received if the surveys were sent via email and sent back to the researcher at the participant's convenience. Another option could have been to conduct a written survey at the beginning or the end of a class with the instructor's permission. Another limitation was that the futility policy used as an example was from a children's hospital; this did influence responses to some of the questions. Given that the students interviewed were in an adult-gerontology program, using an example from an adult hospital might have been more appropriate.

The goal of the project was to evaluate nurses' attitudess of a hospital-based futility policy. The project increases awareness regarding the issues nurses face when caring for terminally ill patients seeking all life-sustaining treatments. The general public is often misled by medical shows that are seen on television. Real life code situations do not bring a loved one back to baseline functioning as portrayed on television. Patients and family members with little-to-no medical background are often pursuing all the technologically savvy treatments available to keep their loved ones alive, which may not be the most appropriate decision. Nurses serve a vital role in education and communication with patients and their families. Nurses may be able to better serve patients and their families if there is a hospital futility policy in place to better support these end of life conversations with patients and families. Nurses in this sample were open to a hospital futility policy in a hospital as a way to provide more support and legal 
backing when caring for terminally ill patients. In addition, ethics committees are underused by the participants of this interview and need to be more accessible to nurses.

Next, recommendations and implications for advanced practice nursing will be discussed. 


\section{Recommendations and Implications for Advanced Practice}

This study presents opportunity for the introduction of a futility policy into hospitals that currently do not have one. Advanced practice registered nurses (APRNs) could be key in conducting a needs assessment, building interdisciplinary support, and in writing, and implementing, a futility policy. The APRN could survey the staff for input as to what they perceive as needed to be included in the futility policy, which would increase staff support and willingness to support the policy. The clinical nurse specialist (CNS) is in a pivotal position to lead resulting practice changes and to monitor the impact on outcomes associated with a futility policy. The CNS can work closely with the staff nurses to provide education and forums to discuss the new policy and the impact it will have on nursing practice. The CNS can also role model and guide the medical team, providing education and serving as a resource for practice change. Nurses and all prescribing providers would require education about a futility policy and how it can change or affect their practice. Most importantly, all healthcare providers would need education on how to utilize the futility policy as well as on how to consult the ethics committee. Measures to promote any member of the healthcare team to request an ethics consult should be initiated.

In order for the futility policy to effectively function in a facility, the rationale for and an understanding of futility policies would need to be supported by the staff. Examples of futility policies from other hospitals could be presented and discussed. Members of these hospitals' ethics committees who have experience utilizing a futility policy could be invited to discuss with staff how a futility policy can impact practice.

There are opportunities for interdisciplinary collaboration. The APRN can participate in patient care meetings to guide and support other practitioners who are involved in the patient's plan of care. The APRN can guide the healthcare team in utilizing the futility policy and also supporting the patient and family through the process. The interdisciplinary staff should incorporate all of the healthcare team including but not 
limited to spiritual care, nurses, physical and occupational therapists, case managers, and social workers. Members of the interdisciplinary team should be involved in the development and dissemination of education forums that may be effectively lead by the APRN. It would be vital for an APRN to be a member of the ethics committee along with other multidisciplinary team members. The APRN could use the futility policy in his or her practice and role model for others.

There are definitely some ethical considerations to consider when employing a futility policy. The ethics committee would need to be not only interdisciplinary, but be comprised of a diverse group of individuals in order to consult appropriately on these cases. Some providers may disagree with the need to a futility policy and may continue to offer all medical interventions regardless of effectiveness due to their own personal beliefs. These beliefs would need to be represented on the ethics committee in order to provide an adequate case discussion. It would be essential for the patient, the family, and the interdisciplinary team parties to be represented in any case in which the futility policy was utilized. All parties should be given an opportunity to speak in front of the ethics committee, which would provide global points of view. The main goal of a futility policy is to advocate for the patient, which is a key point for all patients and healthcare providers to understand.

As a CNS utilizing the Synergy Model, it is important to examine how the futility policy affects the system, nurses and staff, and the patient. The CNS could interview healthcare providers about how the futility policy has impacted their practice. Research could be conducted on how the system is impacted when a futility policy is implemented. Research could also be performed on the cost of medically inappropriate interventions. Readmissions could also be examined to see if there is a decrease, especially for those patients with chronic, progressive conditions. Research could be conducted to evaluate patients' understanding on how a futility policy may impact his or her care, and if it changes how he or she will seek healthcare. Patients and families could be given the 
opportunity to speak about how their care has been impacted since implementation of a futility policy.

The policy could be expanded beyond one facility, which could have significant impact in a small state like Rhode Island. In comparison, a much larger state like Texas has already moved a futility policy into law. It would be interesting to conduct studies with hospitals in Texas to evaluate the impact the Texas Advance Directives Act has had on practice and patient care. In contrast, it would be valuable to study the neighboring states of Texas to evaluate their perceptions of a futility policy. If more states adopt a futility law, there may be a push to make it a national policy. A national futility policy could be championed by the Affordable Care Act due to its potential impact on decreased spending during the last few months of life. The aging population is continuing to increase, therefore, it will be a necessity to avoid utilizing resources on futile care in order to decrease spending. A national futility policy may be seen in the future, after much ethical debate. 


\section{References}

American Nurses Association (2015). Code of Ethics. Nursing World. Retrieved from http://www.nursingworld.org/MainMenuCategories/EthicsStandards/CodeofEthic sforNurses/Code-of-Ethics.pdf

Burns, J.P., \& Truog, R.D. (2007). Futility a concept of evolution. Chest, 132, 19871992.

Clark, A.P. (2010). A model for ethical decision making in cases of patient futility. Clinical Nurse Specialist, 24, 189-190.

Cohen, E. (2007). Fight over baby's life divides ethicists. CNN. Retrieved from http://www.cnn.com/2007/HEALTH/04/25/baby.emilio/

Epstein, E.G. \& Delgado, S. (2010). Understanding and addressing moral distress. The Online Journal of Issues in Nursing, 15, 3.

Ferrell, B. (2006). Understanding the moral distress of nurses witnessing medically futile care. Oncology Nursing Forum, 30, 922-930.

Honeybul, S., Gillett, G.R., \& Ho, K. (2013). Futility in neurosurgery: a patient centered approach. Neurosurgery, 73, 917-922.

Jacobs, B.B. \& Taylor, C. (2005). Medical futility in the natural attitude. Advances in Nursing Science, 28, 288-305.

Lachman, V.D. (2010). Clinical ethics committees: organizational support for ethical practice. MedSurg Nursing, 19, 351-353.

Luce, J.M. (2010). A history of resolving conflicts over end-of-life care in intensive care units in the United States. Critical Care Medicine, 28, 1623-1629.

Mohammed, S. \& Peter, E. (2009). Rituals, death, and the moral practice of medical futility. Nursing Ethics, 16, 292-302.

Nogler, A.F. (2014). Hoping for the best, preparing for the worst. Dimensions of Critical Care Nursing, 33, 22-27. 
Parse, R.R. (1992). Human becoming: Parse's theory of nursing. Nursing Science Quarterly, 5, 35-42.

Quill, T.E. (2005). Terri Schiavo: A tragedy compounded. New England Journal of Medicine, 352, 1630-1633.

Rubin, E. \& Courtwright, A. (2013). Medical futility procedures. What more do we need to know? Chest, $144,1707-1711$.

Rushton, C.H., Kaylor, B.D. \& Christopher, M. (2012). Twenty years since Cruzan and the Patient Self Determination Act: opportunities for improving care at the end of life in critical care settings. AACN Advanced Critical Care, 23, 99-106.

Sibbald, R., Downar, J. \& Hawryluck, L. (2007). Perceptions of "futile care" among caregivers in intensive care units. Canadian Medical Association Journal, 177, 1201-1208.

Terra, S.M. \& Powell, S.K. (2012). Is a determination of medical futility ethical? Professional Case Management, 17, 103-106.

Whitmer, M., Hurst, S., Prins, M., Shepard, K., \& McVey, D. (2009). Medical futility a paradigm as old as Hippocrates. Dimensions of Critical Care Nursing, 28, 67-71.

Wilkinson, D.J.C. \& Savulescu, J. (2011). Knowing when to stop: futility in the ICU. Current Opinion in Anesthesiology, 24, 160-165.

Zier, L.S., Burack, J.H., Micco, G., Chipman, A.K., Frank, J.A. \& White, D.B. (2009). Surrogate decision makers' responses to physicians' predictions of medical futility. Chest, 136, 110-117. 
Appendix A

Case Study

A 58 year old female presents to the emergency room with fever, chills, and cough. She was diagnosed with pneumonia and admitted. Her labs revealed acute renal failure, possibly caused by dehydration. The dehydration was due to new onset diarrhea that was Guiac positive. She had a non-healing right foot ulcer. Her past medical history was significant for non-insulin dependent diabetes, hypertension, hyperlipidemia, peripheral vascular disease, and chronic leg ulcers. She had a $7^{\text {th }}$ grade level education and exhibited childish behaviors such as imitating the IV pump beeping or blocking her ears and screaming. She lived with her brother, and her mother lived in the same apartment building. Both supported all of her decisions for treatment. During her hospitalization, the pneumonia was treated and resolved, but her gastrointestinal (GI) bleed began to worsen. Her lowest recorded hemoglobin was 4.9 (normal is 12-15.5). Her kidney failure also began to worsen. She agreed to partial treatment for the GI bleed and acute renal failure, refused to have a colonoscopy to find and treat the source of the bleed, but agreed to receive a total of 19 units of blood, refused dialysis, but was treated with a bicarbonate drip for metabolic acidosis, received multiple doses of Kayexalate for elevated potassium levels, and received red blood cell boosters. Her non-healing right foot ulcer was debrided twice, although it did not stop bleeding because she had reduced clotting mechanisms. Psychiatry was consulted due to her poor decision-making skills and bizarre behaviors. The patient was, however, deemed competent by the psychiatry team. Patients have a right to refuse care, though choosing partial treatment made it difficult to care for her. The nurses caring for her suffered from frustration and moral distress because administering all this blood to a patient that would not treat the source of the bleed was futile. After months of being admitted and partially treated, the patient agreed to no longer receive blood and made herself "comfort measures only". She was transferred to a nursing home and passed away days later. 


\section{Appendix B \\ Participation Request Letter}

Dear Graduate Student,

My name is Christie Bowser, and I am currently in the graduate nursing program at RI College. I will be conducting interviews as part of my data collection for my Master's Project. I am emailing you to request your participation in a face to face interview to share your thoughts/feelings about a hospital based medical futility policy.

The 1:1 interview will take place on campus at RI College. I will schedule an interview appointment at a time of your convenience. Prior to the interview, you will be given a definition of "futility" for the purpose of this project. You will also be provided with an example of a hospital based futility policy. During the interview, you will be asked several questions, and your answers will be paraphrased as you speak. Your answers will be read back to you by the interviewer to ensure accuracy. The interview is expected to last approximately $30-45$ minutes in total.

When you respond to this email, you will first be asked which facility you are currently employed. Before the interview begins, you will be given an informed consent letter to review and sign, then we will proceed with the interview.

Each interview participant in the research project will be entered to win a $\$ 25$ Panera gift card. One Panera gift card will be awarded from the eligible participants. I thank you in advance for your participation.

Sincerely,

Christie Bowser BSN, RN-BC 
Appendix C

\title{
CONSENT DOCUMENT \\ Rhode Island College
}

\author{
Exploring Nurses' Attitudes About a Futility Policy
}

You are being asked to be in a research study about how you feel about a hospital-based futility policy. You are being asked because you are a graduate nursing student at Rhode Island College with some personal experience in end-of-life care.

Christie Bowser, a graduate nursing student at Rhode Island College, is doing this study.

\section{Why this Study is Being Done (Purpose)}

I am doing this study to explore nurses' attitudes regarding a hospital-based futility policy.

What You Will Have to Do (Procedures)

If you choose to participate in the study, I will ask you to:

- Respond to an email to set up a face to face interview

- Set up an interview date and time at Rhode Island College during the summer at your convenience

- I will give you definitions of moral distress, futility, and an example of a futility policy for your review

- I will ask for demographic data regarding; years of experience as an $\mathrm{RN}$, and the name of the hospital facility where you are employed

- I will ask about 10 questions about the topic of futility, I (the researcher) will record written notes of your answers, and read them back to you for validation

- The interview is projected to take about 30-45 minutes

You Will Be Paid (Compensation)

There will be no financial compensation for participation. As a way to thank you for your time, you will be entered into a raffle for a \$25 Panera Gift Card.

\section{Risks or Discomforts}

This study has minimal risk. You may find that answering some questions is upsetting. We think it would be similar to the kinds of things you talk about with fellow colleagues. You can skip any questions you don't want to answer, and you can stop the interview at any time. If you want to talk to someone about your feelings or about any emotional response to the study questions, you will be provided with the contact information for the Rhode Island College counseling services, which is free of charge to all students.

\section{Benefits of Being in the Study}

There is no direct benefit from participation in this study. 


\section{Deciding Whether to Be in the Study}

Being in the study is your choice to make. Nobody can force you to be in the study. You can choose not to participate in the study, and nobody will hold it against you. You can change your mind and quit the study at any time, and you do not have to give a reason. If you decide to quit later, nobody will hold it against you.

\section{How Your Information will be Protected}

Because this is a research study, results will be summarized across all participants and shared in reports that we publish and presentations that we give. Your name will not be used in any reports. We will take several steps to protect the information you give us so that you cannot be identified. The information will be kept in a locked office file, and seen only by myself and other researchers who work with me. The only time I would have to share information from the study is if it is subpoenaed by a court, or if you are suspected of harming yourself or others, then I would have to report it to the appropriate authorities. Also, if there are problems with the study, the records may be viewed by the Rhode Island College review board responsible for protecting the rights and safety of people who participate in research. The information will be kept for a minimum of three years after the study is over, after which time it will be destroyed.

\section{Who to Contact}

You can ask any questions you have now. If you have any questions later, you can contact me at candrade_2522@email.ric.edu or (401)524-9462.

If you think you were treated badly in this study, have complaints, or would like to talk to someone other than the researcher about your rights or safety as a research participant, please contact Christine Marco at IRB@ric.edu, by phone at 401-456-8598.

You will be given a copy of this form to keep. 


\section{Statement of Consent}

I have read and understand the information above. I am choosing to participate in the study "Exploring nurses' attitudes about a hospital-based futility policy." I can change my mind and quit at any time, and I don't have to give a reason. I have been given answers to the questions I asked, or I will contact the researcher with any questions that come up later. I am at least 18 years of age.

Print Name of Participant:

Signature of Participant: Date:

Name of Researcher Obtaining Consent: 


\section{Appendix D}

\section{Interview}

Futility: Treatment or clinical interventions that are not likely to result in benefit to the patient or produce the expected outcome (Terra \& Powell, 2012). 


\section{Appendix E}

\section{Boston Children's Futility Policy}

Boston Children's Futility Policy Key Elements (Burns \& Truog, 2007):

1. The policy is "bilateral": addresses the claims of overtreatment by the clinicians and families. The clinicians or the family can request an ethics consult.

2. There must be clear documentation of efforts to achieve resolution with the patient and family, emphasizing that limiting the use of life-sustaining treatments will not lead to abandonment.

3. If repeated efforts fail, then the case is referred to the institutional Ethics Advisory Committee for a three-phase decision process:

a. Meeting with the committee and clinical team to present the medical perspective on the case

b. Meeting with the committee and the patient or family to "tell their story"

c. Meeting of the committee alone to make a decision

4. If the committee supports the caregivers' assessment, the four options are

a. Hospital administration could request the clinicians to pursue further attempts with the patient or family

b. The physician and hospital could attempt to transfer care

c. Hospital administration could seek a judicial resolution to the conflict, on grounds that the patient's surrogate is not acting in the patient's best interest.

d. Hospital administration could sanction the unilateral foregoing or removal of life-sustaining treatments. 


\section{Appendix F}

Interview

Interview questions:

1. Please describe to me your understanding of medical futility.

2. What is your experience with futile care? How does it affect you in both your personal and professional life?

3. In the literature, it is argued that medical futility is a way of rationing healthcare. Do you agree with this statement? Can you describe to me what you feel each one means?

4. Does the hospital you work in have an ethics committee? Do you have any experiences with utilizing the ethics committee? Can you describe that experience? Do you feel comfortable requesting an ethics consult?

5. How do you feel a futility policy would function in a hospital? How do you feel it would affect your practice?

6. Are you familiar with moral distress? Can you describe your feelings about moral distress? Can you reflect on a patient experience you've had that you still think about?

7. Do you feel the presence of a futility policy would increase or decrease moral distress?

8. What do you think about Boston Children's Hospital futility policy I have given you? Would that deter you from working there? Why or why not? How do you feel it would affect futile care?

9. How do you feel a futility policy would work in a hospital? How would it change your practice? 